\title{
Ground state phase diagram of twisted three-leg spin tube in magnetic field
}

\author{
Kouki Yonaga* and Naokazu Shibata \\ Department of Physics, Tohoku University, Sendai, 980-8578, Japan
}

\begin{abstract}
We study the ground state phase diagram of the twisted three-leg spin tube in magnetic fields by the density matrix renormalization group (DMRG) method. The twisted spin tube is composed of triangular unit cells and possesses strong quantum fluctuations under geometrical frustration. We apply the sine square deformation method to remove strong boundary effects and obtain smooth magnetization curves without steps of finite systems. With the analysis of the magnetization curves and correlation functions we determine the ground state phase diagram consisting of (a) a Tomonaga-Luttinger (TL) liquid characterized by spin- $\frac{3}{2}$ Heisenberg model, (b) 3-sublattice state named UUD with $1 / 3$ magnetization and (c) TL-liquid of massless chirality with 1/3 magnetization plateau, (d) TL-liquid of massless spin mode with or without chirality quasi long-range order.
\end{abstract}

(a)

\section{Introduction}

Following Anderson's proposal of a spin liquid on 2D triangular lattice, ${ }^{1)}$ frustrated quantum spin systems have been studied to find novel quantum phenomena. Now it is widely believed that geometrical frustration leads to degenerate ground states and gives rise to diverse low energy properties of quantum systems. So called "chirality" defined by cross product of spins on a triangular lattice is a new degree of freedom emerged in such systems and the interplay between spin and chirality is an interesting research topic of frustrated systems. Here we apply external magnetic field and study the ground-state properties described by the spin and the chirality under the magnetic field. Since magnetic field introduces unidirectional anisotropy only in spin space, it generally suppresses spin fluctuations while leaves the chirality unchanged that will lead to a new class of quantum state.

In this paper we investigate the twisted three-leg spin tube consisting of triangular unit cells as a typical 1D fully frustrated quantum spin system (Fig. 1). ${ }^{2-4)}$ The ground state of this model has been studied mainly in several limiting cases. In the limit of weakly interacting triangles, where intratriangle interaction written in the red (thick) lines in Fig. 1 (a) are stronger than the other interactions written in the black (thin) lines, Fouet et al. have shown that the ground state is spin-chirality dimer state with translational symmetry breaking. ${ }^{5-8)}$ In the opposite limit of strongly interacting triangles, they have pointed out that the effective model is a spin- $\frac{3}{2}$ Heisenberg model with a gapless excitation mode. Thus, it is expected that the ground state is characterized either by a spin-chirality dimer or a spin- $\frac{3}{2}$ quasi long-range-order, and a first order phase transition separates them. ${ }^{5,9)}$

In magnetic field, Fouet et al. found 1/3 magnetization plateau in the region of weakly interacting triangles. ${ }^{5)}$ When the intra- and inter-coupling of the triangles are comparable, Chen et al. have reported that the ground state has 3sublattice structure and UUD state appears in 1/3 magnetization plateau. ${ }^{10)}$ In this plateau, Plat et al. have shown that the chirality behaves as XY pseudospin in the limit of weakly interacting triangles ${ }^{11)}$ suggesting the chirality mode is gapless while the spin mode is gapful in this limit. Although twisted

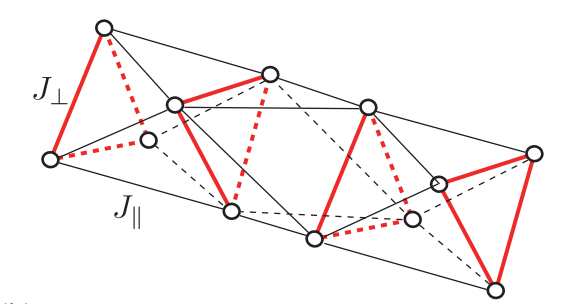

(b)

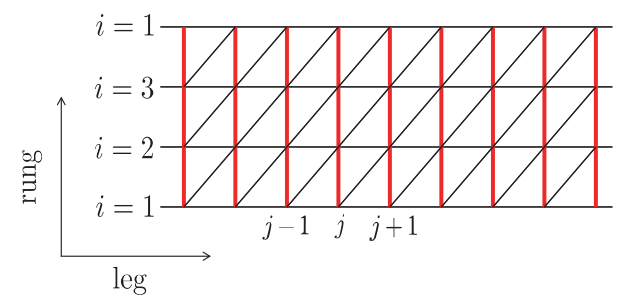

Fig. 1. (Color online) (a) Structure of twisted three-leg spin tube. (b) Equivalent structure of the spin tube with the periodic boundary condition in the direction of rung.

three-leg spin tube is expected to have rich phases, e.g. 3sublattice states, two-component Tomonaga-Luttinger liquid of the spin and chirality, and 1/3 magnetization plateau, detailed ground-state phase diagram has not yet been clarified.

In this paper, we apply the density matrix renormalization group (DMRG) method ${ }^{12)}$ with the sine square deformation $(\mathrm{SSD})^{13)}$ and determine the ground state phase diagram of the twisted three-leg spin tube in magnetic field. The paper is organized as follows. In $\S 2$, we define the Hamiltonian of the twisted spin tube and explain recently developed technique of SSD. In $\$ 3$ and 4 our numerical results are presented and analyzed to determine the ground state phase diagram. We summarize our results in $\$ 5$.

\section{Model and Method}

The Hamiltonian we studied here is defined by

$$
H=J_{\perp} \sum_{j=1} \sum_{i=1}^{3} \boldsymbol{S}_{i, j} \cdot \boldsymbol{S}_{i+1, j}
$$


(a)

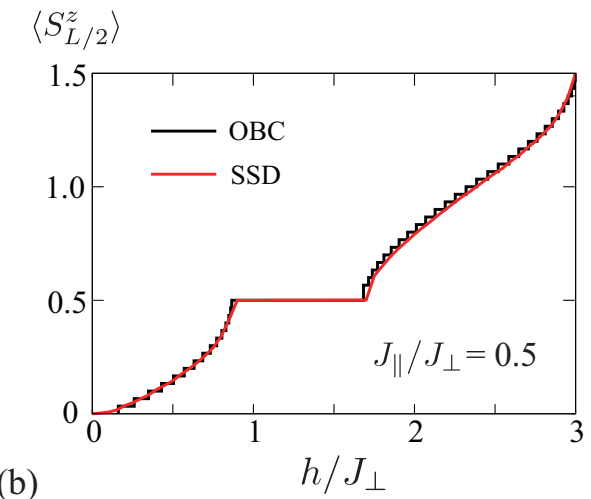

(b)

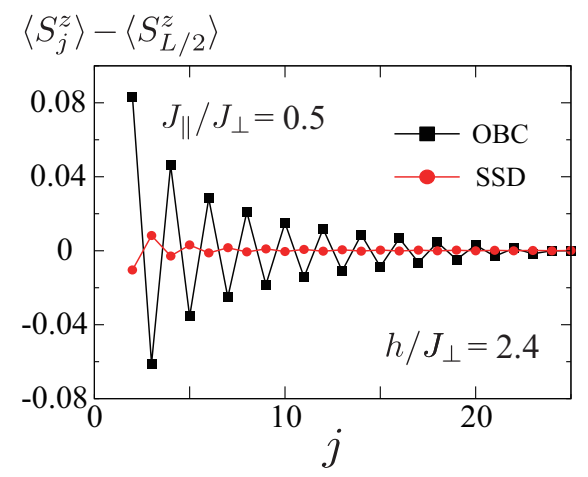

Fig. 2. (Color online) Local magnetization at $J_{\|} / J_{\perp}=0.5$ obtained by DMRG under the usual open boundary condition (OBC) and SSD; (a) local magnetization at the center of the system $\left\langle S_{L / 2}^{z}\right\rangle=\sum_{i=1}^{3}\left\langle S_{i, L / 2}^{z}\right\rangle$ and (b) site dependence of the local magnetization $\left\langle S_{j}^{z}\right\rangle-\left\langle S_{L / 2}^{z}\right\rangle$ at $h / J_{\perp}=2.4$. Total number of unit triangles $L$ is 60 and the typical truncation error in DMRG is $10^{-6}$.

$$
\begin{aligned}
& +\quad \boldsymbol{J}_{\|} \sum_{j=1} \sum_{i=1}^{3} \boldsymbol{S}_{i, j} \cdot \boldsymbol{S}_{i, j+1}+\boldsymbol{S}_{i, j} \cdot \boldsymbol{S}_{i+1, j+1} \\
& -\quad h \sum_{j=1} \sum_{i=1}^{3} S_{i, j}^{z}
\end{aligned}
$$

where $S_{i, j}$ represents spin- $\frac{1}{2}$ operator at rung $i$ and leg $j$, and $J_{\perp}\left(J_{\|}\right)$is antiferromagnetic exchange coupling of intra (inter) triangles. The last term is Zeeman energy with $h$ being the external magnetic field. To diagonalize this Hamiltonian we use the DMRG method, which is usually applied to open boundary conditions $(\mathrm{OBC}) .{ }^{12)}$ However, spins at the ends of the open system cause artificial effects called "boundary effects" that sometimes make it difficult to study the bulk properties of the system. Recently, Gendiar et al. introduced the SSD and succeed in removing boundary effects. ${ }^{13-15)}$ The SSD is a kind of energy scale deformation defined as

$$
H_{S S D}=\sum_{j=1}^{L} f_{0}(j) h_{0}(j)+\sum_{j=1}^{L-1} f_{1}(j) h_{1}(j, j+1),
$$

where $h_{0}(j)$ is on-site term corresponding to the first and third term in Eq.(1) and $h_{1}(j, j+1)$ is the nearest neighbor interaction term such as the second term in Eq.(1). $f_{l}(j)$ is the scaling function and defined as

$$
f_{l}(j)=\sin ^{2}\left[\frac{\pi}{L}\left(j+\frac{l-1}{2}\right)\right] .
$$

Since the energy scale near the edges of the system is neg- (a)

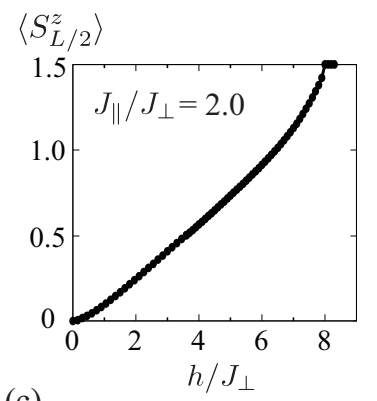

(b)

(c)
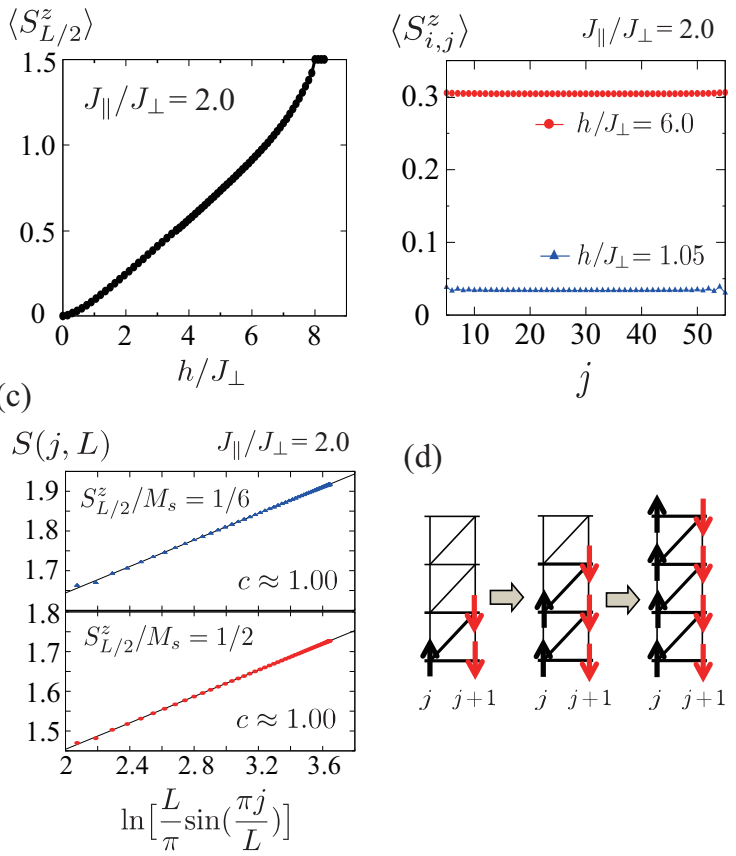

(d)

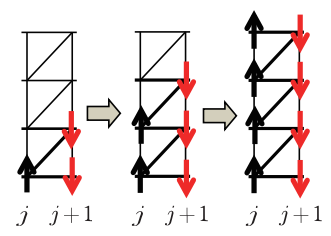

Fig. 3. (Color online) Results at $J_{\|} / J_{\perp}=2.0$ obtained by DMRG: (a) magnetization curve $\left\langle S_{L / 2}^{z}\right\rangle$, (b) local magnetization $\left\langle S_{i, j}^{z}\right\rangle$ and (c) entanglement entropy $S(j, L)$ of block size $j$ in the $L$ site system under usual OBC. The upper (lower) panel in (c) shows the result for $S_{L / 2}^{z} / M_{S}=1 / 6\left(S_{z} / M_{s}=1 / 2\right)$. (d) Dominant local spin structure in the limit of $J_{\|} / J_{\perp} \gg 1.0$.

ligibly small, the boundary effects are efficiently suppressed around the center of the system where the scaling function is order unity. The bulk properties of the model is then evaluated around the center of the system. Figures 2 (a) and (b) show the magnetization curve of $\left\langle S_{L / 2}^{z}\right\rangle=\sum_{i}^{3}\left\langle S_{i, L / 2}^{z}\right\rangle$ and the real space profile of the local magnetization $\left\langle S_{j}^{z}\right\rangle=\sum_{i=1}^{3}\left\langle S_{i, j}^{z}\right\rangle$. Without the SSD, oscillation of $\left\langle S_{j}^{z}\right\rangle$ extends into the central part of the system and the averaged magnetization is discretized by the conservation of the total $S_{j}^{z}$. In contrast, the oscillation under SSD is clearly suppressed and the magnetization curve is obtained as a smooth function of $h$. Such removing of the boundary effects and the smooth response to the external field are important features of the SSD and these make it easy to find anomalies in the response to the external field such as magnetization plateaus. The SSD was originally used to restore the translational invariance in 1D free fermion system and it has been shown that the ground state of critical systems such as XXZ model and (extend) Hubbard model obtained under SSD is identical to the one under the periodic boundary condition (PBC). ${ }^{14-19)}$ In this study we use the SSD for the analysis of the ground state correlation functions and magnetization curves.

In our work, we consider the unit triangle as a single site and keep up to 1200 basis states in the DMRG calculation whose truncation error is the order of $10^{-6} \sim 10^{-8}$. We show mainly the numerical results of the finite system of size $L=60$. Since the numerical results obtained near the edges of the system are meaningless in SSD, we only use the results around the center. 
(a)

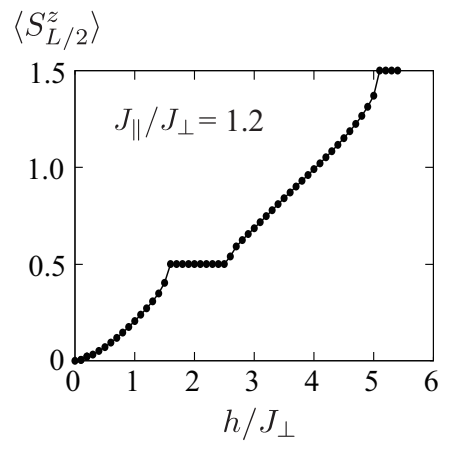

(d)

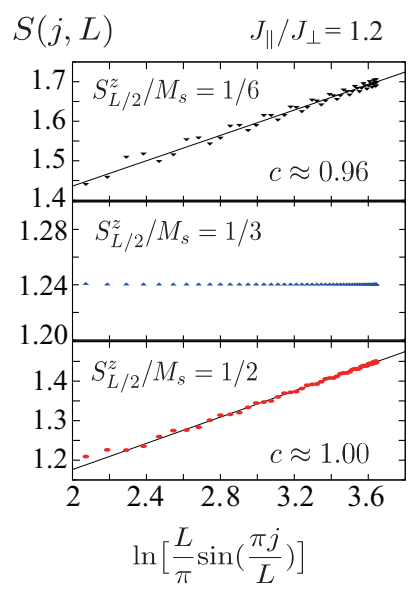

(b)

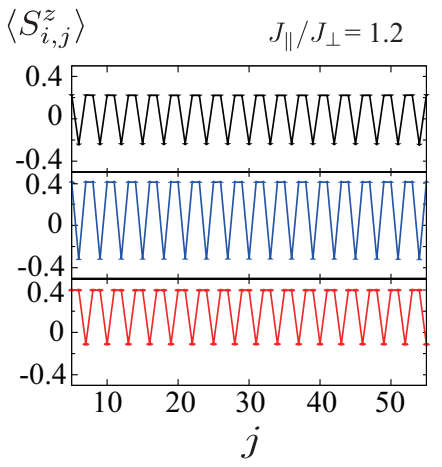

(e)
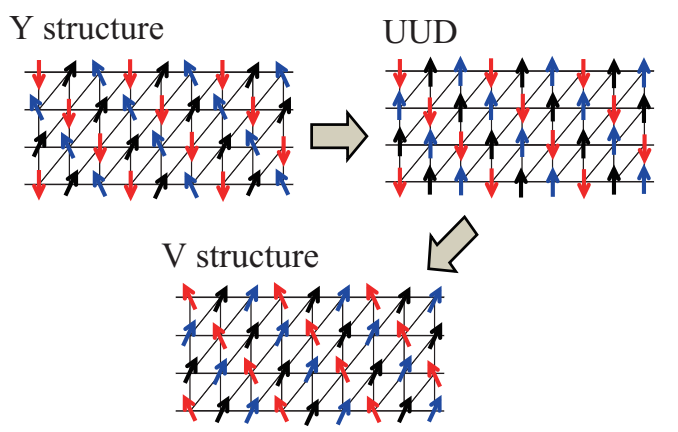

(c)

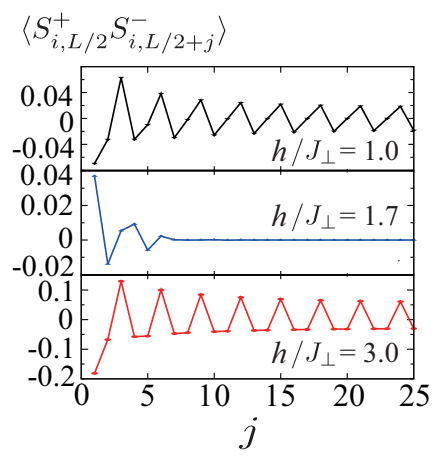

Fig. 4. (Color online) Results at $J_{\|} / J_{\perp}=1.2$ obtained by DMRG: (a) magnetization $\left\langle S_{L / 2}^{z}\right\rangle$, (b) site dependence of local magnetization $\left\langle S_{i, j}^{z}\right\rangle$, (c) transverse spin correlation $\left\langle S_{i, L / 2}^{+} S_{i, L / 2+j}^{-}\right\rangle$and (d) entanglement entropy $S(j, L)$ at $S_{L / 2}^{z} / M_{S}=1 / 6,1 / 3$ and $1 / 2$. The upper, middle and lower panels in (b) and (c) correspond to $h / J_{\perp}=1.0,1.7$ and 3.0, respectively. (e) schematic spin structures of Y, UUD and V states.

\section{Results}

\section{$3.1 \quad J_{\|} / J_{\perp} \gtrsim 1.0$}

We start from the case of $J_{\|} / J_{\perp} \gtrsim 1.0$, where strong intertriangle antiferromagnetic couplings ferromagnetically align the spins in intra-triangles as shown in Fig.3 (d). The effective Hamiltonian is then described by spin- $\frac{3}{2}$ Heisenberg model, ${ }^{5)}$ whose low-energy properties are characterized by a Tomonaga-Luttinger (TL) liquid. As shown in Fig.3 (a) the magnetization at $J_{\|} / J_{\perp}=2.0$ monotonically increases with the increase in magnetic field and the local magnetization (a)

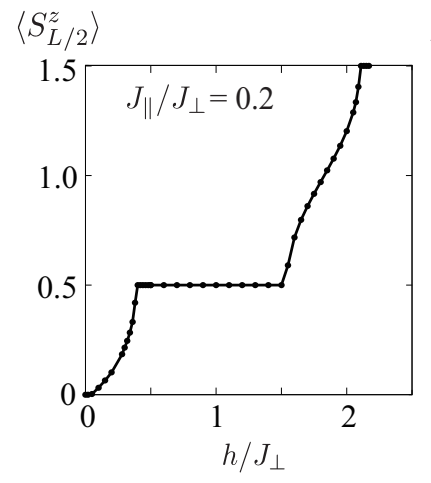

(b)

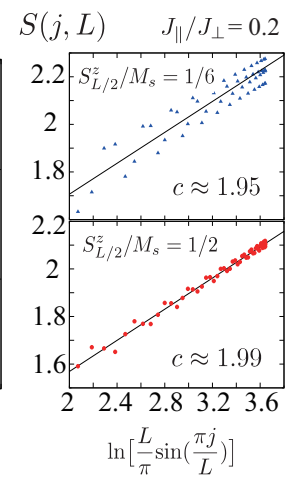

Fig. 5. (Color online) DMRG results at $J_{\|} / J_{\perp}=0.2$; (a) magnetization curve and (b) entanglement entropy $S(j, L)$ at $S_{L / 2}^{z} / M_{s}=1 / 6$ and $1 / 2$. $\left\langle S_{i, j}^{z}\right\rangle$ is site-independent. To confirm this gapless state we calculate the size dependence of the entanglement entropy which is analytically obtained for TL liquid as

$$
S(j, L)=s_{B} \ln \left[\frac{L}{\pi} \sin \left(\frac{\pi j}{L}\right)\right]
$$

where $j$ and $L$ are the block and the total system size, and $s_{B}$ is the constant given by the central charge $c$ as $s_{B}=c / 6(c / 3)$ for OBC (PBC). Since the above formula is obtained without $\mathrm{SSD}$, we calculate $S(j, L)$ under usual OBC without SSD. Figure 3 (c) shows $S(j, L)$ at $S_{L / 2}^{z} / M_{s}=1 / 6$ and $1 / 2$, where $M_{s}$ is the saturation magnetization per unit triangle, $3 / 2$. We find the central charge $c$ is close to 1 in both cases that indicates the ground state is characterized by one-component TomonagaLuttinger liquid (TLL1) in agreement with the previous work by Fouet et al.

\section{$3.2 J_{\|} / J_{\perp} \approx 1.0$}

We next consider the intermediate region $J_{\|} / J_{\perp} \approx 1.0$. Figure 4 (a) shows the magnetization curve at $J_{\|} / J_{\perp}=1.2$ where we find clear $1 / 3$ magnetization plateau which divides the ground state into three phases; below $1 / 3$ magnetization plateau, on the plateau, above the plateau. The upper panel in Fig.4 (b) shows the local magnetization $\left\langle S_{i, j}^{z}\right\rangle$ of the ground state below the plateau. The local magnetization $\left\langle S_{i, j}^{z}\right\rangle$ shows the presence of the 3-sublattice structure. As shown in the upper panel of Fig.4 (c), the transverse correlation function $\left\langle S_{i, L / 2}^{+} S_{i, L / 2+j}^{-}\right\rangle$has 3 -sites period. To under- 
(a)
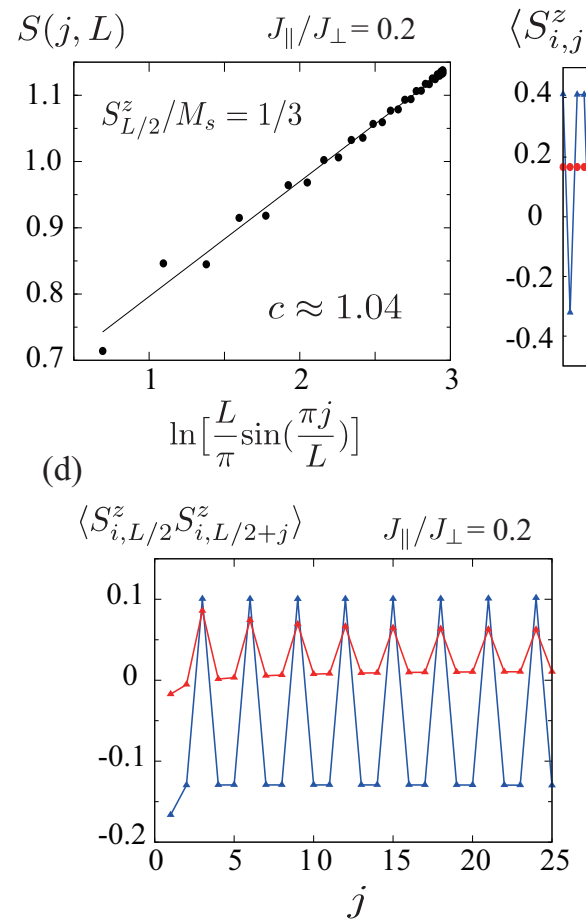

(b)
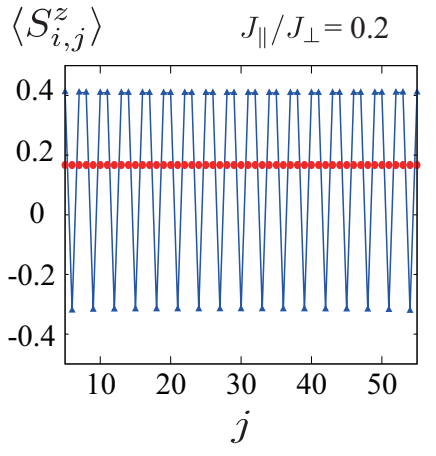

(e) (c)

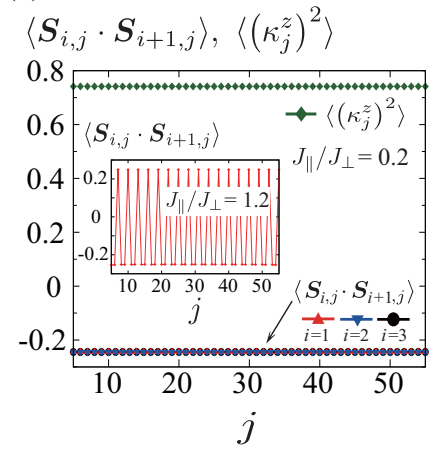

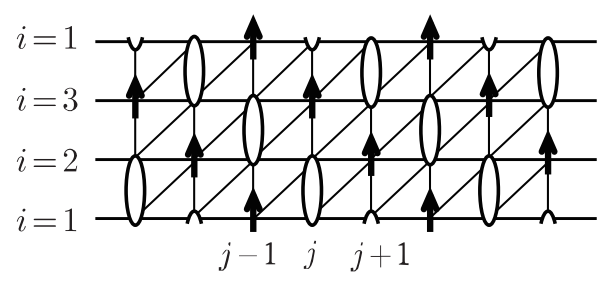

Fig. 6. (Color online) DMRG results in $1 / 3$ plateau at $J_{\|} / J_{\perp}=0.2$; (a) entanglement entropy $S(j, L)$, (b) local magnetization $\left\langle S_{i, j}^{z}\right\rangle$, (c) local singlet correlation $\left\langle\boldsymbol{S}_{i, j} \cdot \boldsymbol{S}_{i+1, j}\right\rangle$ and the z-component of the local chirality $\left\langle\left(\kappa_{j}^{z}\right)^{2}\right\rangle$, (d) longitudinal spin correlation $\left\langle S_{i, L / 2}^{z} S_{i, L / 2+j}^{z}\right\rangle$ and (e) schematic spin structure in the $1 / 3$ magnetization plateau at $J_{\|} / J_{\perp} \lesssim 1.0$. In (a), (b) and (d), we also plot the results at $J_{\|} / J_{\perp}=1.2$ for comparison. The inset in (c) represents local spin correlation $\left\langle\boldsymbol{S}_{i, j} \cdot \boldsymbol{S}_{i+1, j}\right\rangle$ for UUD structure at $J_{\|} / J_{\perp}=1.2$.

stand this spin structure let us consider the classical limit. When $\left\langle S_{i, L / 2}^{+} S_{i, L / 2+j}^{-}\right\rangle$is large and positive, $x y$ component of the two spins at $L / 2$ and $(L / 2+j)$ sites is parallel, while it is almost orthogonal when $\left\langle S_{i, L / 2}^{+} S_{i, L / 2+j}^{-}\right\rangle \sim 0$, and antiparallel when $\left\langle S_{i, L / 2}^{+} S_{i, L / 2+j}^{-}\right\rangle<0$. This simple picture indicates that the ground state below $1 / 3$ plateau has Y structure, which is a deformation of $120^{\circ}$ state, where two spins are oriented upward to the $z$ direction with anitferromagnetic correlations in $x y$ components while one spin is antiparallel to $z$-direction as shown in Fig.4 (e). Similarly we can see that the ground state above $1 / 3$ plateau is characterized by $\mathrm{V}$ structure shown in Fig.4 (e). As is shown in the power law decay of the correlation function $\left\langle S_{i, L / 2}^{+} S_{i, L / 2+j}^{-}\right\rangle$, the ground states of $\mathrm{Y}$ and $\mathrm{V}$ structures are expected to have massless spin excitations consistent with Mermin-Wagner theorem. This is confirmed by the entanglement entropy $S(j, L)$ shown in the upper and lower panels of Fig.4 (d), whose $j$ dependence corresponds to $c=1$ one-component TL liquid.

In contract to the above results, $1 / 3$ plateau state called UUD has short range correlation as shown in the middle panel of Fig.4 (c). The local magnetization $\left\langle S_{i, j}^{z}\right\rangle$ in Fig.4 (b) has clear 3-sublattice structure with squeezed moments due to quantum fluctuations. The entanglement entropy $S(j, L)$ is independent of $j$ for large $j$ showing the ground state at $1 / 3$ plateau is not critical consistent with the short range correlation functions.

All the 3-sublattice structures Y, UUD and V are originated from $120^{\circ}$ classical state. This was first pointed out by Chubukov in $2 \mathrm{D}$ triangle lattice. ${ }^{20-24)}$ They have explained these 3-sublattice structures are stabilized by quantum fluctuations. We think the similar 3-sublattice structures in the twisted spin tube are also stabilized by the same mechanism since the unit cell of the twisted spin tube is identical to that of the $2 \mathrm{D}$ triangular lattice when $J_{\|} / J_{\perp}=1.0$.

\section{$3.3 J_{\|} / J_{\perp} \lesssim 1.0$}

We finally investigate the ground state in the region of weakly interacting triangles $J_{\|} / J_{\perp} \lesssim 1.0$. Although the magnetization curve in Fig.5 (a) shows $1 / 3$ plateau at $J_{\|} / J_{\perp}=0.2$, the spin structure in this plateau is different from UUD found at $J_{\|} / J_{\perp} \approx 1.0$ as will be shown in the following. In the limit of weakly interacting triangles, the effective Hamiltonian is written as

$$
\begin{aligned}
H_{\mathrm{eff}} & =\frac{2 J_{\|}}{3} \sum_{j} \boldsymbol{S}_{j} \cdot \boldsymbol{S}_{j+1} \\
& +\frac{4 J_{\|}}{3} \sum_{j} \boldsymbol{S}_{j} \cdot \boldsymbol{S}_{j+1}\left(\tau_{j}^{+} \tau_{j+1}^{-}+\text {h.c. }\right) \\
& -h \sum_{j=1} S_{j}^{z},
\end{aligned}
$$

where $\tau_{j}^{+}\left(\tau_{j}^{-}\right)$represents the raising (lowering) operator acting on the two chirality states $\left|\tau_{z}=+1 / 2\right\rangle$ and $\left|\tau_{z}=-1 / 2\right\rangle$ of the unit triangle at $j$-th site, and $\boldsymbol{S}_{j}$ is spin- $\frac{1}{2}$ operator of the same unit triangle. ${ }^{5,7,8,25)}$ Below the magnetization plateau, the bosonization analysis shows that the spin and chirality degrees of freedoms are separated within a perturbation analysis with respect to the second term in Eq. $5 .^{26-29)}$ To confirm this result we first calculate the entanglement entropy $S(j, L)$. As seen in the upper panel of Fig. 5 (b), $S(j, L)$ has linear size dependence on $\ln \left[\frac{L}{\pi} \sin \frac{\pi j}{L}\right]$ and the central charge $c$ is close to 2 . 
This value of the central charge means the ground state below $1 / 3$ magnetization plateau is characterized by two-component Tomonaga Luttinger liquid (TLL2). ${ }^{30,31)}$ Similar result is also obtained above the magnetization plateau as shown in the lower panel of Fig. 5 (b). We therefore conclude that the elementary excitation above and below $1 / 3$ plateau have two massless excitation modes for the spin and chirality.

We next see $j$-dependence of $S(j, L)$ in $1 / 3$ plateau. In general, the entanglement entropy becomes constant for large $j$ if all the excitations from the ground state have a finite gap. As seen in Fig.6 (a), $S(j, L)$ at $J_{\|} / J_{\perp}=0.2$ has linear dependence on $\ln \left[\frac{L}{\pi} \sin \frac{\pi j}{L}\right]$ with a constant $c$ close to 1 . This result indicates that the elementary excitation has one massless mode. Since the spin excitation has a gap in the magnetization plateau, the low energy massless excitation of Eq. 5 is described by chirality ${ }^{11)}$ whose Hamiltonian is

$$
H_{\text {eff }}=\frac{J_{\|}}{6} \sum_{j}\left[1+2\left(\tau_{j}^{+} \tau_{j+1}^{-}+\text {h.c. }\right)\right] .
$$

We therefore conclude that the ground state is characterized by TLL1 of a chirality XY model consistent with the previous work by Plat $e t$ al. ${ }^{11)}$

The local spin magnetization $\left\langle S_{i, j}^{z}\right\rangle$ of the above ground state is shown in Fig. 6 (b). In contract to UUD state at $J_{\|} / J_{\perp} \approx 1.0$, the local magnetization at $J_{\|} / J_{\perp}=0.2$ is uniform. The uniform ground state is also shown in the spin correlation function $\left\langle\boldsymbol{S}_{i, j} \cdot \boldsymbol{S}_{i+1, j}\right\rangle$ in the same triangle and the local chirality expectation value $\left\langle\left(\kappa_{j}^{z}\right)^{2}\right\rangle$, where $\kappa^{z}$ is given by

$$
\kappa_{j}^{z}=\sum_{i=1}^{3}\left(\boldsymbol{S}_{i, j} \times \boldsymbol{S}_{i+1, j}\right)^{z} .
$$

As shown in Fig. 6 (c), $\left\langle\boldsymbol{S}_{i, j} \cdot \boldsymbol{S}_{i+1, j}\right\rangle \approx-0.25$ and $\left\langle\left(\kappa_{j}^{z}\right)^{2}\right\rangle \approx$ 0.75 . These results show the coexistence of a spin singlet and chirality in the unit triangle. This is contrasted with the results at $J_{\|} / J_{\perp}=1.2$ where UUD structure appears with small $\left\langle\left(\kappa^{z}\right)^{2}\right\rangle$ and one positive and two negative local spin correlations, $\left\langle S_{i, L / 2}^{z} S_{i, L / 2+j}^{z}\right\rangle$, within a triangle. This difference in the local correlation makes clear difference in the long range spin correlation $\left\langle S_{i, L / 2}^{z} S_{i, L / 2+j}^{z}\right\rangle$ shown in Fig. 6 (d) that is positive or nearly 0 at $J_{\|} / J_{\perp}=0.2$, while it has a structure of 3-site period with one positive and two negative at $J_{\|} / J_{\perp}=1.2$. We illustrate the schematic diagram of the ground state at $J_{\|} / J_{\perp}=0.2$ in Fig.6 (e). The ground state is a superposition of one singlet and one polarized spin like 0.5-0-0 in the direction of both rung and leg. Since each polarized spin is connected to spin singlet states, there is no penalty in the exchange energy among them. Therefor $\left\langle S_{L / 2}^{z} S_{L / 2+j}^{z}\right\rangle$ has 3-site period while $\left\langle S_{j}^{z}\right\rangle$ is uniform. This is caused by strong geometrical frustration and a unique characteristic of the twisted spin tube.

\section{Discussion}

The phase boundaries of the ground state obtained in each region $J_{\|} / J_{\perp} \gtrsim 1.0, J_{\|} / J_{\perp} \approx 1.0$ and $J_{\|} / J_{\perp} \lesssim 1.0$ are presented in the phase diagram shown in Fig.7 (a). The boundary is determined by the appearance or the change of 3 sublattice structure and the central charge of TL liquids. For the boundary between $\mathrm{V}$ and TL liquid states in the region of $J_{\|} / J_{\perp}>1.0$, we have used the appearance of the 3 sublattice (a)

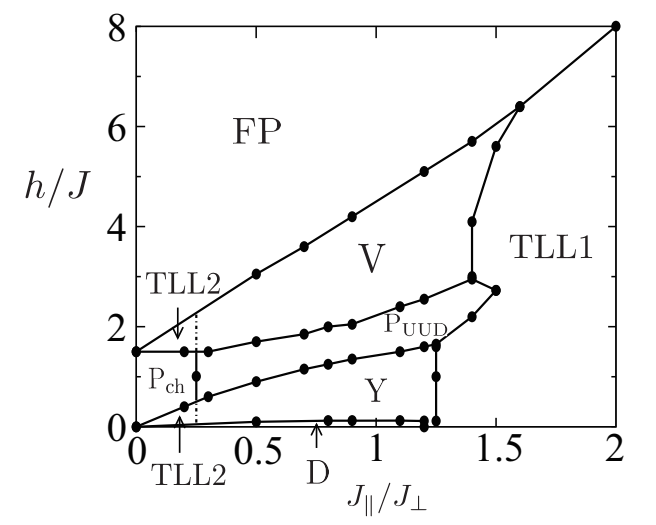

(b)

(c)
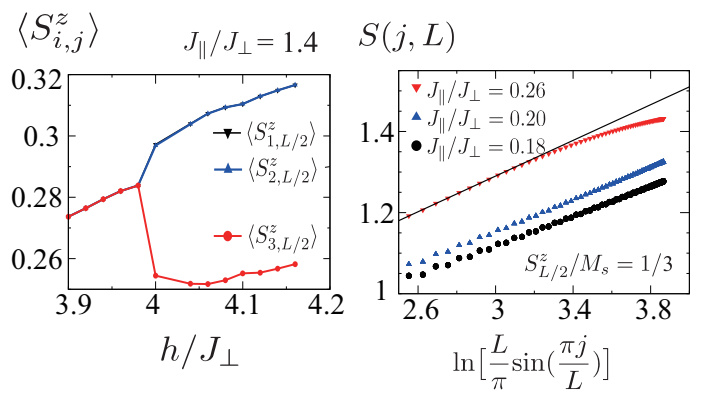

Fig. 7. (Color online) (a) Ground state phase diagram of the twisted spin tube. The labels D, TLL1, TLL2 represent the dimer state, one- and twocomponent Tomonaga-Luttinger liquid, respectively. The labels $\mathrm{Y}$ and V represent $\mathrm{Y}, \mathrm{V}$ states. $\mathrm{P}_{\mathrm{UUD}}$ and $\mathrm{P}_{\mathrm{ch}}$ corresponds to $1 / 3$ magnetization plateau with UUD structure and $1 / 3$ plateau with TLL1 of chirality, respectively. FP is fully polarized state. (b) local magnetization $\left\langle S_{i, L / 2}^{z}\right\rangle$ at $J_{\|} / J_{\perp}=1.4$ as a function of $h / J_{\perp}$. (c) entanglement entropy $S(j, L)$ at $S_{L / 2}^{z} / M_{s}=1 / 3$.

structure as a signal of the transition. This is clearly shown in the local spin polarization $\left\langle S_{i, L / 2}^{z}\right\rangle$ at $J_{\|} / J_{\perp}=1.4$. As shown in Fig.7 (b), $\left\langle S_{i, L / 2}^{z}\right\rangle$ splits into two values at $h \approx 4$ which corresponds to the change from spin- $\frac{3}{2}$ TLL1 to V state. The phase diagram around the plateau state is rather complicated. Since the corresponding 2D triangular lattice does not have $1 / 3$ plateau in the region of $J_{\|} / J_{\perp} \lesssim 0.5,{ }^{24)}$ the appearance of the magnetization plateau with massless mode of chirality is a unique feature of the twisted spin tube. Above and below the magnetic plateau, the elementary excitations at $J_{\|} / J_{\perp} \lesssim 0.3$ are characterized by the central charge $c \approx 2$ and are different from 3-sublattice state and TLL1.

Figure 7 (c) shows $S(j, L)$ for different $J_{\|} / J_{\perp}$ in $1 / 3$ magnetization plateau. It is seen that $S(j, L)$ has linear size dependence on $\ln \left[\frac{L}{\pi} \sin \frac{\pi j}{L}\right]$ at $J_{\|} / J_{\perp}=0.18$ and 0.20 , while it dose not at $J_{\|} / J_{\perp}=0.26$. Thus, TLL1 of the chirality with uniform magnetization extends to $J_{\|} / J_{\perp} \approx 0.26$. We expect the transition from TLL1 of the chirality to UUD state is second order because we find no signal of level crossing, but it is difficult to confirm this point within a finite system. Detailed finite size scaling analysis is needed to clarify the nature of the transition.

\section{Summary}

We have studied the ground state of the twisted three-leg spin tube in magnetic field by the DMRG with the SSD and identified various phases such as TLL1, TLL2, 3-sublattice 
state and $1 / 3$ magnetization plateau. In particular we have confirmed that for $J_{\|} / J_{\perp} \lesssim 0.3$ the ground state in the $1 / 3$ plateau has low energy excitations described by massless mode of chirality. The coexistence of the spin and chirality degrees of freedom and the interplay between them are characteristic features of twisted three-leg spin tube and the origin of its diverse ground states.

\section{Acknowledgment}

K. Y. would like to thank Y. Fuji for helpful advice.This work was supported by Grants-in-Aid for Scientific Research (No. 26400344) from MEXT Japan.

1) P. W. Anderson, Mater. Res. Bull. 8 (1973) 153.

2) J. Schnack, H. Nojiri, P. Kögerler, G. J. T. Cooper and L. Cronin, Phys. Rev. B 70 (2004) 1774420.

3) N. B. Ivanov, J. Schnack, R. Schnalle, J. Richter, P. Kögerler, G. N. Newton, L. Cronin, Y. Oshima and H. Nojiri, Phy. Rev. Lett. 105 (2010) 037206.

4) Y. Furukawa, Y. Sumida, K. Kumagai, F. Borsa, H. Nojiri, Y. Shimizu, H. Amitsuka, K. Tenya, P. Kögerler and L. Cronin, J. Conf. Ser. 320 (2011) 012047.

5) J.-B. Fouet, A. Läuchli, S. Pilgram, R. M. Noack and F. Mila, Phys. Rev. B 73 (2006) 014409.

6) A. Lüscher, R. M. Noack, G. Misguich, V. N. Kotov and F. Mila, Phys. Rev. B 70 (2004) 060405(R).

7) H. J. Schulz, cond-mat/9605075.

8) K. Kawano and M. Takahashi, J. Phys. Soc. Jpn. 66 (1997) 4001.

9) K. Okunishi, S. Yoshikawa, T. Sakai and S. Miyashita, Int. J. Mod. Phys.
C 29 (2009) 1423.

10) Ru Chen, Hyejin Ju, Hong-Chen Jiang, O. A. Starykh and L. Balents, Phys. Rev. B 87 (2013) 165123.

11) X. Plat, S. Capponi and P. Pujol, Phys. Rev. B 85 (2012) 174423.

12) S. R. White, Phys. Rev. Lett. 69 (1992) 2863; S.R. White, Phys. Rev. B 48 (1993) 10345.

13) A. Gendiar, R. Krcmar and T. Nishino, Prog. Theor. Phys. 122 (2009) 953123 (2010) 393.

14) T. Hikihara and T. Nishino, Phys. Rev. 83 (2011) 060414(R).

15) C. Hotta and N. Shibata, Phys. Rev. B 86 (2012) 041108(R).

16) H. Katsura, J. Phys. A, Math. Theor. 44 (2011) 252001.

17) H. Katsura, J. Phys. A, Math. Theor. 45 (2012) 115003.

18) A. Gendiar, M. Daniška, Y. Lee and T. Nishino, Phys. Rev. A 83 (2011) 0522118 .

19) N. Shibata and C. Hotta, Phys. Rev. B 84 (2011) 115116.

20) A. V. Chubukov and D. I. Golosov, J. Phys. Condens. Matter 3 (1991) 69.

21) S. Miyashita and H. Shiba, J. Phys. Soc. Jpn. 53 (1984) 1145.

22) H. Kawamura and S. Miyashita, J. Phys. Soc. Jpn. 53 (1984) 9.

23) C. Griest, S. Head, J. Alicea and O. A. Starykh, Phys. Rev. B 84 (2011) 245108.

24) T. Coletta, M. E. Zhitomirsky and F. Mila, Phys. Rev. B 87 (2013) 060407(R).

25) Y. Fuji, S. Nishimoto, H. Nakada and M. Oshikawa, Phys. Rev. B 89 (2014) 054425.

26) E. Orignac, R. Citro and N. Andrei, Phys. Rev. B 61 (2000) 11533.

27) R. Citro, E Orignac, N Andrei and S Qin, J. Phys. Condens. Matter 12 (2000) 3041.

28) Kunj Tandon, Siddhartha Lal, Swapan K. Pati, S. Ramasesha and Diptiman Sen, Phys. Rev. B 59395.

29) D. C. Cabra, A. Honecker and P. Pujol, Phys. Rev. B 58 (1998) 6241.

30) M. Sato and T. Sakai, Phys. Rev. B 75 (2007) 014411.

31) M. Sato, Phys. Rev. B 75 (2007) 174407. 